\title{
XIX. Ueber die Bestimmung der Positionen von Punkten und Ebenen nach einer Drehung um einen bestimmten Winkel und um eine bekannte Axe.
}

\author{
Von
}

R. W. H. T. Hudson B. A. in Cambridge.

(Mit 1 Textfigur.)

\section{Rechtwinkelige Axen.}

Man nehme drei zu einander senkrechte Bezugsaxen $O X, O Y, O Z$. Betreffs der positiven Richtung der Drehung um irgend eine Linic ist eine Ucbereinkunft zu treffen, und die gewöhnlich angenommene ist die folgende: eine positive Drehung um $O X$ führt $O Y$ nach $O Z$ hin, ein solche um $O Y$. lührt $O Z$ nach $O X$ hin und eine um $O Z$ führt $O X$ nach $O Y$ hin. Um den positiven Sinn der Drehung für irgend eine Linie $O N \mathrm{zu}$ finden, muss man eine Bewegung von $O N$ annehmen, wodurch sie mit einer der Axen zur Deckung gelangt, z. B. mit $O Z$, und dann die vorhergehende Regel anwenden.

Nimmt man die Axen, wie in der Figur angegeben, in welcher $O X$ auf den Beobachter zulaufend gedacht ist, so bewirkt

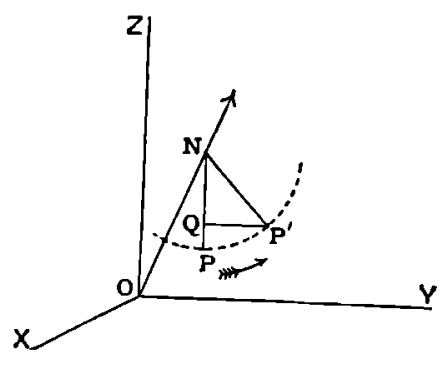
eine positive Drehung um irgend eine Linie $O N$, zusammen mit einer Schiebung parallel $O N$, eine Rechtsschraubung. Wenn die Axen $O X$ und $O Y$ vertauscht würden, so würde die Schraubung eine linkc werden.

Die Figur stellt die Ortsveründerung eines Punktes $P$ in die Lage $P^{\prime}$ dar, welche durch Drehung um einen Winkel $\varphi$ um $O N$ in positiver Richtung bewirkt wird. Aufgabe ist, die Coordinaten $\left(x^{\prime} y^{\prime} x^{\prime}\right)$ von $P^{\prime}$ als Function derjenigen von $P(x y \approx), \varphi$ und der gegebenen Richtung $O N$ zu bestimmen.

Es sei $\angle N O X=\lambda, \angle N O Y=\mu, \angle N O Z=\nu$. 
$P N, P^{\prime} N$ sind senkrecht zu $O N$ und $\angle P N P^{\prime}=\rho$. $P^{\prime} Q$ ist senkrecht zu $N P$.

Um $N O$ zu finden, bemerken wir, dass es die Projection von $O P$ auf $O N$ ist. Diher:

$$
\begin{aligned}
N O=O P \cos P O N= & O P\left\{\begin{array}{l}
x \\
O P \\
\cos \lambda+\stackrel{y}{O P} \cos \mu+\frac{z}{O P} \cos \nu
\end{array}\right\} \\
& =x \cos \lambda+y \cos \mu+z \cos \nu .
\end{aligned}
$$

Es ist geeignet die Coordinaten von $N$ zu henutzen; diese sind $\xi, \eta, \zeta$, wo

$$
\begin{aligned}
& \xi=O N \cos \lambda=(x \cos \lambda+y \cos \mu+z \cos \nu) \cos \lambda \\
& \eta=O N \cos \mu=(x \cos \lambda+y \cos \mu+z \cos \nu) \cos \mu \\
& \zeta=O N \cos \nu=(x \cos \lambda+y \cos \mu+z \cos \nu) \cos \nu .
\end{aligned}
$$

Die Richtung von $Q P^{\prime}$ ist gesucht.

Die Richtungscosinus von $O N$ sind $\cos \lambda, \cos \mu, \cos \nu$.

$$
\text { - } N P-\frac{x-\xi}{N P}, \frac{y-\eta}{N P}, \frac{z-\zeta}{N P},
$$

wofür gesetzt werden möge

$p$,

$q$,

$r$.

Daher sind diejenigen von $Q P^{\prime}$, welche senkrecht zu beiden sind: $\pm(r \cos \mu-q \cos \nu), \pm(p \cos \nu-r \cos \lambda), \pm(q \cos \lambda-p \cos \mu)$.

Das Vorzeichen wird in jedem Falle bestimmt dadurch, dass, wenn $O N$ durch die Bewegung mit $O Z$ zusammenfült, und $N P$ in der Richtung $O X$ sich bewegt, alsdann $Q P^{\prime}$ in die Richtung $O Y$ gelangt, d. h., wenn $\cos \lambda=0=\cos \mu, \cos \nu=1, p=1, q=0=r$, müssen wir erhalten:

$$
\pm(p \cos \nu-r \cos \lambda)=1 \text {. }
$$

Es muss daher das obere Zeichen genommen werden.

Wir sind jetzt in der Lage, die Coordinaten von $P^{\prime}$ niederzuschreiben, weil wir die Lïngen und Richtungen von $O N, N Q, Q P^{\prime}$ kennen.

Nämlich:

$$
\left.\begin{array}{ll} 
& x^{\prime}-x=(1-\cos \varphi)(\xi-x)+\sin \varphi(z \cos \mu-y \cos \nu) \\
\text { ebenso } & y^{\prime}-y=(1-\cos \varphi)(\eta-y)+\sin \varphi(x \cos \nu-z \cos \lambda) \\
\text { und } & z^{\prime}-\approx=(1-\cos \varphi)(\zeta-z)+\sin \varphi(y \cos \lambda-x \cos \mu)
\end{array}\right\}
$$

$$
\begin{aligned}
x^{\prime} & =O N \cos \lambda+N Q \cdot p+Q P^{\prime}(r \cos \mu-q \cos \nu) \\
& =\xi+N P^{\prime} \cos \varphi \cdot \frac{x-\xi}{N P}+N P^{\prime} \sin \varphi(r \cos \mu-q \cos \nu) \\
& =\xi+\cos \varphi(x-\xi)+\sin \varphi(z \cos \mu-y \cos \nu)
\end{aligned}
$$

oder:

worin $\xi, \eta, \zeta$ die oben gefundenen Werthe haben.

Die Relationen (2), welche die neue Position eines beliebigen Punktes angeben, sind in Minchin's Statik 1889, 2, 103 entwickelt, und wir sind oben auch seiner Methode der Beweisführung gefolgt. 
Wenn $x, y, z$ als Functionen von $x^{\prime}, y^{\prime}, z^{\prime}$ gesucht werden, so ist nur nöthig, in den obigen Formeln $x$ und $x^{\prime}$ etc. zu vertauschen und das Zeichen von $\varphi \mathrm{zu}$ wechseln.

$\Lambda$ ngenommen, es sei gesucht die neue Position einer Ebene

$$
h x / a+k y / b+l z / c=1 .
$$

Nun ist der Punkt $h / a, k / b, l / c$ der Pol $P$ dieser Ebene in Bezug auf die Sphäre

$$
x^{2}+y^{2}+z^{2}=1 \text {, }
$$

und die Relation von Pol und Polare bleibt unverïndert bei einer beliebigen Rotation um $O$. Daher ist die neue Position der Ebene

$$
h^{\prime} x / a+k^{\prime} y / b+l^{\prime} z / c=1,
$$

wo $h_{i}^{\prime} a, k^{\prime} / b, l^{\prime} / c$ die Coordinaten der neuen Position $P^{\prime}$ des ursprünglichen Poles $P$ sind. Dieselben werden daher aus (2) erhalten, und sind:

$$
\left.\begin{array}{l}
\left.\frac{h^{\prime}}{a}=\frac{h}{a} \cos \varphi+O N \cos \lambda(1-\cos \varphi)+\sin \varphi\left(\frac{l}{c} \cos \mu-\frac{l}{b} \cos \nu\right)\right) \\
\left.\frac{k^{\prime}}{b}=\frac{k}{b} \cos \varphi+O N \cos \mu(1-\cos \varphi)+\sin \varphi\left(\frac{h}{a} \cos \nu-\frac{l}{c} \cos \lambda\right)\right),(;) \\
\frac{l^{\prime}}{c}={ }_{c}^{l} \cdot \cos \varphi+O N \cos \nu(1-\cos \varphi)+\sin \varphi\left(\frac{l}{b} \cos \lambda-\frac{h}{a} \cos u\right)
\end{array}\right)
$$

\section{Schiefo Axen.}

Man nehme rechtwinkelige $\Lambda$ xen wie im Falle (1) und gebe den auf sie hezogenen schiefen $\Lambda$ xen die Richtungscosinus $\left(l_{1} m_{1} n_{1}\right),\left(l_{2} m_{2} n_{2}\right),\left(l_{3} m_{3} n_{3}\right)$.

$O P$ bilde mit den schiefen $\Lambda \mathrm{xen}$ die Winkel $\theta_{1}, \theta_{2}, \theta_{3}$, dann ist

$$
\cos \theta_{i}=\left(l_{i} x+m_{i} y+n_{i} z\right) \div O P,(i=1,2,3) \text {. }
$$

Gesucht sind die Winkel $\theta_{1}{ }^{\prime}, \theta_{2}{ }^{\prime}, \theta_{3}{ }^{\prime}$, welche $O P^{\prime}$ mit den schiefen dxen bildet. Wir haben

$$
\cos \theta_{i}^{\prime}=\left(l_{i} x^{\prime}+m_{i} y^{\prime}+n_{i} z^{\prime}\right) \div O P^{\prime},(i=1,2,3) .
$$

Durch Multiplication der Formel (2) mit $\frac{l}{O P}$ resp. $-\frac{m}{O P}, \frac{n}{O P}$ (unter Weglassung des Index) und $\Lambda$ ddition erhält man daher:

$$
\cos \theta^{\prime}=\cos \varphi \cos \theta+(1-\cos \varphi) \frac{l \xi+m \eta+n \zeta}{O P}+\sin \varphi\left|\begin{array}{ccc}
l & m & n \\
\cos \lambda & \cos \mu & \cos \nu \\
\frac{x}{O P} & \frac{y}{O P} & \frac{z}{O P}
\end{array}\right| .
$$

Nun ist, wenn die Rotationsaxe $O N$ mit den schiefen $\Lambda$ xen die Winkel $\omega_{1}, \omega_{2}, \omega_{3}$ bildet: 


$$
\begin{gathered}
\cos \omega_{i}=l_{i} \underset{O N}{\xi}+m_{i} \frac{\eta}{O N}+n_{i} \frac{\zeta}{O N},(i=1,2,3), \\
\therefore \cos \theta_{i}^{\prime}=\cos \varphi \cos \theta_{i}+(1-\cos \varphi) \cos P O N \cos \left(\omega_{i}+\sin \varphi A_{i} .\right.
\end{gathered}
$$
nanten

Um den Werth von $\Delta_{1}$ zu erhalten, multipliciren wir die Determi-

$$
\left|\begin{array}{ccc}
l_{1} & m_{1} & n_{1} \\
\cos \lambda & \cos \mu & \cos \nu \\
x & y & z \\
O P & \overline{O P} & \overline{O P}
\end{array}\right| \text { und }\left|\begin{array}{ccc}
l_{1} & m_{1} & n_{1} \\
l_{2} & m_{2} & n_{2} \\
l_{3} & m_{3} & n_{3}
\end{array}\right|
$$

mit einander, und erhalten

$$
\left|\begin{array}{ccc}
1 & \cos \gamma & \cos \beta \\
\cos \omega_{1} & \cos \omega_{2} & \cos \omega_{3} \\
\cos \theta_{1} & \cos \theta_{2} & \cos \theta_{3}
\end{array}\right|
$$

Nun ist das Quadrat der zweiten Determinantc

$$
\left|\begin{array}{ccc}
1 & \cos \gamma & \cos \beta \\
\cos \gamma & 1 & \cos \alpha \\
\cos \beta & \cos \alpha & 1
\end{array}\right|,
$$

dies möge glcich $D$ gesetzt werden.

Folglich ist

$$
\Delta_{1} D^{\frac{1}{2}}=\left|\begin{array}{ccc}
1 & \cos \gamma & \cos \beta \\
\cos \omega_{1} & \cos \omega_{2} & \cos \omega_{3} \\
\cos \theta_{1} & \cos \theta_{2} & \cos \theta_{3}
\end{array}\right|,
$$

in ähnlicher Weise können $\Delta_{2}$ und $\Delta_{3}$ bestimmt werden.

Es erübrigt nur, $\cos P O N$ aus $\omega_{1}, \omega_{2}, \omega_{3}, \theta_{1}, \theta_{2}, \theta_{3}$ und den Winkeln zwischen den schiefen Axen $\alpha, \beta, \gamma$ zu berechnen.

Es seien $e, f, g$ die Richtungsverhältnisse von $O P$.

Man construire ein Parallelepiped, dessen Diagonale $O P$ und dessen Kanten den schiefen $\Lambda$ xen parallel sind, und projicire auf $O N$, so erhält man :

$$
\cos P O N=e \cos \omega_{1}+f \cos \omega_{2}+g \cos \omega_{3} ;
$$

ferner, wenn man auf die schiefen Axen projicirt, ergiebt sich:

$$
\begin{aligned}
& \cos \theta_{1}=e+f \cos \gamma+g \cos \beta \\
& \cos \theta_{2}=e \cos \gamma+f+g \cos \alpha \\
& \cos \theta_{3}=e \cos \beta+f \cos \alpha+g .
\end{aligned}
$$

Durch Elimination von $e, f, g$ erhält man

$$
\therefore\left|\begin{array}{cccc}
\cos P O N & \cos \omega_{1} & \cos \omega_{2} & \cos \omega_{3} \\
\cos \theta_{1} & 1 & \cos \gamma & \cos \beta \\
\cos \theta_{2} & \cos \gamma & 1 & \cos \alpha \\
\cos \theta_{3} & \cos \beta & \cos \alpha & 1
\end{array}\right|=0
$$

oder 
$\cos \operatorname{PON}\left|\begin{array}{ccc}1 & \cos \gamma & \cos \beta \\ \cos \gamma & 1 & \cos \alpha \\ \cos \beta & \cos \alpha & 1\end{array}\right|+\left|\begin{array}{cccc}1 & \cos \gamma & \cos \beta & \cos \theta_{1} \\ \cos \gamma & 1 & \cos \alpha & \cos \theta_{2} \\ \cos \beta & \cos \alpha & 1 & \cos \theta_{3} \\ \cos \omega_{1} & \cos \omega_{2} & \cos \omega_{3} & 0\end{array}\right|=0$

So ist $\cos P O N$ gefunden und das Resultat kann in folgender Weise zusammengefasst werden:

Wenn die Gerade $O P$, welche mit den $\Lambda$ xen die Winkel $\theta_{1}, \theta_{2}, \theta_{3}$ bildet, durch eine Drehung um den Winkel $\varphi$ um die Gerade $O N$, welche mit den Axen die Winkel $\omega_{1}, \omega_{2}, \omega_{3}$ bildet, in die Position $O P^{\prime}$, welche die Richtungswinkel $\theta_{1}{ }^{\prime}, \theta_{2}{ }^{\prime}, \theta_{3}{ }^{\prime}$ besitzt, übergeführt wird, so ist:

$\cos \theta_{i}^{\prime}=\cos \varphi \cos \theta_{i}+(1-\cos \varphi) \cos P O N \cos \omega_{i}+\sin \varphi \Delta_{i}, \quad(i=1,2,3)$.

I'm die neuc l'osition einer belieljigen Ebene zu finden, bemerken wir, dass die Richtungscosinus der Normalen zu der Ebene $h \frac{x}{a}+k \frac{y}{b}+l \frac{z}{c}=1$ sind: $\varrho \frac{h}{a}, \varrho \frac{k}{b^{-}}, \varrho \frac{l}{c}$, wo $\varrho$, die bei der Drehung unverändert bleibt, cine Senkrechte aus $O$ auf dic Ebene ist.

Jie gesuchten Formeln sind daher:

$$
\left.\begin{array}{l}
\begin{array}{l}
h^{\prime} \\
a
\end{array}=\frac{h}{a} \cos \varphi+(1-\cos \varphi) \frac{\cos P O N}{\varrho} \cos \omega_{1}+\sin \varphi D_{1} \\
k^{\prime} \\
\ddot{b}=\frac{k}{b} \cos \varphi+(1-\cos \varphi) \frac{\cos P O N}{\varrho} \cos \omega_{2}+\sin \varphi D_{2} \\
\frac{l^{\prime}}{c}=\frac{l}{e} \cos \varphi+(1-\cos \varphi) \frac{\cos P O N}{\varrho} \cos \omega_{3}+\sin \varphi D_{3}
\end{array}\right\},
$$

wo

$$
-\frac{\cos I^{\prime} O N}{\varrho}=
$$

$$
\begin{gathered}
\left|\begin{array}{cccc}
1 & \cos \gamma & \cos \beta & h_{/} / a \\
\cos \gamma & 1 & \cos \alpha & k / b \\
\cos \beta & \cos \alpha & 1 & l / c \\
\cos \omega_{1} & \cos \omega_{2} & \cos \omega_{3} & 0
\end{array}\right| \div\left|\begin{array}{ccc}
1 & \cos \gamma & \cos \beta \\
\cos \gamma & 1 & \cos \alpha \\
\cos \beta & \cos \alpha & 1
\end{array}\right| ; \\
D_{1}=D^{-\frac{1}{2}}\left|\begin{array}{ccc}
1 & \cos \gamma & \cos \beta \\
\cos \omega_{1} & \cos \omega_{2} & \cos \omega_{3} \\
h_{i}^{\prime} a & k_{i}^{\prime} b & l / c
\end{array}\right|, \text { etc. }
\end{gathered}
$$

Wenn der l)rehungswinkel $180^{\circ}$ ist, können die T'ransformationsformeln direct in folgender WVeise erhalten werden.

Da der Punkt $N$ der Mittelpunkt der Geraden $P P^{\prime}$ ist, so ist seinc Projection auf jede der $\Lambda$ xen der Mittelpunkt der Projection auf die betreffende $\Lambda x e$ :

$$
\begin{aligned}
& \therefore O P \cos \theta_{i}+O P^{\prime} \cos \theta_{i}^{\prime}=2 O N \cos \omega_{i} ; \\
& \therefore \cos \theta_{i}+\cos \theta_{i}^{\prime}=2 \cos \omega_{i} \cos P O N .
\end{aligned}
$$


344 R. W. H. T. Hudson. Lieber die Bestimmung der Positionen von Punkten etc.

Es möge, wie vorher, die Ehene

$$
h \frac{x}{a}+k \frac{y}{b}+l \frac{z}{c}=1
$$

werden:

$$
h^{\prime} \frac{x}{a}+k^{\prime} \frac{y}{b}+l^{\prime} \stackrel{*}{c}=1 .
$$

Jann müssen wir setzen:

$$
\cos \theta_{1}=\varrho \frac{h}{a}, \text { etc. }, \quad \cos \theta_{1}^{\prime}=\varrho \frac{h^{\prime}}{a}, \text { etc. }
$$

folglich :

$$
\frac{h+h^{\prime}}{a \cos \omega_{1}}=\frac{k+k^{\prime}}{b \cos \omega_{2}}=\frac{l+l^{\prime}}{c \cos \omega_{3}}=2 \frac{\cos P O N}{\varrho},
$$

wovon der Werth in Gleichung (8) gegeben ist. 